\title{
Rapid Molecular Diagnosis of Pulmonary Tuberculosis in Children Using Nasopharyngeal Specimens
}

\author{
Heather J. Zar, ${ }^{1,2}$ Lesley Workman, ${ }^{1,2}$ Washiefa Isaacs, ${ }^{1,2}$ Jacinta Munro, ${ }^{1,2}$ Faye Black, ${ }^{1,2}$ Brian Eley, ${ }^{1,2}$ Veronica Allen, ${ }^{3,4}$ \\ Catharina C. Boehme, ${ }^{5}$ Widaad Zemanay, ${ }^{3,4}$ and Mark P. Nicol ${ }^{3,4}$ \\ ${ }^{1}$ Department of Paediatrics and Child Health, University of Cape Town, ${ }^{2}$ Red Cross War Memorial Children's Hospital, ${ }^{3}$ Division of Medical \\ Microbiology, and ${ }^{4}$ Institute for Infectious Diseases and Molecular Medicine, University of Cape Town, National Health Laboratory Service, \\ Groote Schuur Hospital, Cape Town, South Africa; and ${ }^{5}$ Foundation for Innovative New Diagnostics, Geneva, Switzerland
}

Background. A rapid diagnosis of pediatric pulmonary tuberculosis (PTB) using Xpert MTB/RIF (Mycobacterium tuberculosis/rifampicin) automated testing on induced sputum (IS) is possible, but the capacity for performing IS is limited. The diagnosis using a nasopharyngeal aspirate (NPA), which can be non-invasively obtained, is desirable.

Methods. Paired specimens (NPA and IS) were tested using smear, liquid culture and Xpert. The diagnostic accuracy of Xpert and smear was compared with culture for different specimens in children with suspected PTB.

Results. There were 535 children [median age 19 months, 117 (21.9\%) HIV-infected] who had one IS and one NPA specimen; 396 had two paired specimens. A positive smear, Xpert test or culture occurred in 30 (5.6\%), 81 (15.1\%) and 87 children (16.3\%), respectively. The culture yield was higher from IS (84/87, 96.6\%) vs NPA (61/87, $70.1 \%, P<.001)$. Amongst children with two paired specimens, 63 culture-confirmed cases occurred [60 (95.2\%) IS vs $48(76.2 \%)$ NPA, $P=.002]$. The sensitivity of two Xpert tests was similar for IS and NPAs [(45/63) $71 \%$ vs $(41 / 63) 65 \%, P=.444)]$; the sensitivity of smear was lower for IS $(21 / 63,33 \%)$ and NPA $(16 / 63,25 \%)$. The incremental yield from a second IS was 9 cases $(17.6 \%)$ by culture and $9(25 \%)$ by Xpert testing; a second NPA increased the culture yield by $10(26.3 \%)$ and Xpert by $11(36.7 \%)$. Xpert specificity was $99.1 \%(98.1-100)$ for IS and 98.2\% (96.8-99.6) for NPAs. Xpert testing provided faster results than culture (median 0 vs 15 days, $P<.001$ ).

Conclusions. Xpert testing on 2 NPAs is useful in children with suspected PTB, particularly in settings where IS and culture are not feasible.

A diagnosis of pulmonary tuberculosis (PTB) in children remains challenging due to non-specific clinical or radiological signs and an inability to obtain appropriate specimens [1]. Moreover, as childhood tuberculosis is paucibacillary, a diagnosis using smear microscopy is usually negative. However, recent advances in specimen collection and diagnostic testing have shown that

Received 20 March 2012; accepted 14 June 2012; electronically published 2 July 2012.

Correspondence: Heather J. Zar, Dept of Paediatrics and Child Health, 5th F ICH Bldg, Red Cross War Memorial Children's Hospital, Cape Town, South Africa (heather.zar@uct.ac.za).

Clinical Infectious Diseases 2012:55(8):1088-95

(C) The Author 2012. Published by Oxford University Press on behalf of the Infectious Diseases Society of America. All rights reserved. For Permissions, please e-mail: journals.permissions@oup.com.

DOI: $10.1093 /$ cid/cis598 a microbiologic diagnosis can be effectively made [2]. Repeated induced sputum (IS) specimens are feasible and effective for the culture-confirmed diagnosis of PTB in children [3-5].

We recently reported that a rapid diagnosis of PTB in children using the Xpert MTB/RIF automated, cartridge-based test (Xpert, Cepheid, CA, USA) for Mycobacterium tuberculosis and resistance to rifampicin could be effectively obtained using IS specimens [6]. Two Xpert tests detected 76\% of culture-confirmed cases, twice the yield from smear, with a specificity of $99 \%$ [6]. Rifampicin resistance was reliably detected, and the results were faster than liquid culture [6]. These provide pediatric evidence to support WHO recommendations that Xpert testing replace smear as the initial diagnostic test in suspected HIV-associated tuberculosis or 
multidrug-resistant tuberculosis and as a follow-on test in settings where these are less of a concern [7].

The implementation of pediatric Xpert testing is constrained by the limited capacity to obtain IS. The use of a nasopharyngeal aspirate (NPA) is attractive, as this can be easily and non-invasively obtained with a lower risk of nosocomial transmission. However, a study of Peruvian children comparing 2 NPAs with 2 gastric aspirates (GAs) found that only $50 \%$ of culture-positive cases were detected with NPAs [8]. Moreover, an in-house polymerase chain reaction (PCR) had insufficient sensitivity or specificity to detect tuberculosis for clinical use [8]. However, no comparison with IS was done. A small Ugandan study reported promising results for culture and PCR performed on NPAs from children with suspected tuberculosis [9]. The availability of Xpert now provides a reliable test with a high sensitivity and specificity for IS samples $[6,10]$. We aimed to investigate the yield from repeated NPAs compared to IS specimens using culture, smear and Xpert.

\section{MATERIALS AND METHODS}

Consecutive children hospitalized with suspected PTB in Cape Town, South Africa from 1 February 2009 to 30 June 2011 were enrolled. The reasons for hospitalisation included severe or very severe pneumonia defined by WHO criteria, the need for oxygen or intravenous therapy, or social conditions precluding home care. Children under 15 years were eligible if they had a cough for more than 14 days and if one of the following conditions existed: a household tuberculosis contact within the preceding 3 months, weight loss or failure to gain weight within the preceding 3 months, a positive skin test (TST) to purified protein derivative (PPD 2TU, PPD RT23, Staten Serum Institute, Denmark), or a chest radiograph suggestive of PTB. Children were excluded if they had received tuberculosis drug(s) for longer than 72 hours, didn't live in Cape Town, couldn't attend follow-up visits, if informed consent was unavailable, or if an IS or NPA sample was not obtained.

Routine investigations included chest radiography and a TST and HIV test when the HIV status was unknown. HIVinfected children were classified by WHO clinical staging and CDC immunological classification [11, 12]; the CD4 count and HIV viral load (Abbott RealTime HIV-1 assay, Des Plaines, IL, USA) were measured. Nutritional status was calculated as height-for-age and weight-for-age $\mathrm{z}$ scores using the CDC/WHO 1998 reference standard.

Tuberculosis therapy was initiated at the discretion of the hospital doctor. Follow-up visits were done at 1, 3 and 6 months for children on therapy and at 1 and 3 months for those untreated. The response to treatment was assessed using clinical features and chest radiograph at treatment completion.
Written, informed consent was obtained from a parent or legal guardian. The study was approved by the Ethics Committee of the Faculty of Health Sciences, University of Cape Town.

\section{Nasopharyngeal Aspirate and Sputum Induction}

An NPA sample was obtained before the IS specimen. Two drops of sterile saline were instilled into each nostril and the nasopharynx was suctioned using a sterile catheter with a mucus trap. A second NPA sample was obtained the following day or a minimum of 4 hours after the first IS.

Sputum induction was done at least 30 minutes after an NPA, following a 2-3 hour fast by a trained research nurse as previously described [3]. A second IS sample was obtained the following day or a minimum of 4 hours after the first specimen. Pulse oximetry was performed throughout the procedure and 30 minutes thereafter.

NPA and IS specimens were processed within 2 hours of collection by an accredited laboratory staffed with trained technologists, using standardized protocols. For both specimens, following decontamination with $\mathrm{N}$-acetyl-L-cysteine and sodium hydroxide (1.0\% final concentration), the centrifuged deposits were resuspended in $1.5 \mathrm{~mL}$ of phosphate buffer. A drop of sediment was used for fluorescent acid-fast smear microscopy. For Xpert testing, $1.4 \mathrm{~mL}$ of specimen reagent was added to $0.7 \mathrm{~mL}$ of the resuspended sputum/NPA pellet and processed as previously reported [10]. Automated liquid culture (BACTEC MGIT, Becton Dickinson, USA) was performed using $0.5 \mathrm{~mL}$ of the resuspended pellet. Cultures were incubated for 6 weeks.

Positive cultures were identified by acid-fast staining followed by MTBDRplus testing (Hain Lifesciences, Hehren, Germany) to confirm the presence of $M$. tuberculosis and rifampicin and isoniazid resistance [13]. The staff who performed the Xpert testing were blind to the culture results. If Xpert identified rifampicin resistance, the corresponding cultured isolate also underwent phenotypic resistance testing by automated liquid MGIT (mycobacteria growth indicator tube) culture.

\section{Definition of Tuberculosis Disease}

Children were categorized as "Definite tuberculosis" (any specimen positive for $M$. tuberculosis on culture), "Not tuberculosis" (documented resolution of symptoms and signs at follow-up in children who didn't receive tuberculosis treatment), or "Possible tuberculosis" (all others).

\section{Analysis}

The primary reference standard was a positive culture for M. tuberculosis from an IS or NPA sample. We excluded children without at least one paired IS/NPA specimen or children with extrapulmonary tuberculosis. We separately analysed patients with at least one paired IS and NPA and those with two paired specimens. For the former, we used a positive culture 
from any NPA or IS specimen as the reference standard to determine the sensitivity of a single Xpert test on the first IS or NPA. Since the sensitivity of a single culture is likely to be suboptimal in children, it is likely that true positive Xpert results would be assigned as falsely positive if only a single culture was available for comparison. Therefore, for the analysis of specificity, we included only children with two Xpert and culture results. The sensitivity, specificity and predictive values of the assays with 95\% confidence intervals (95\% CI) were determined. Data were analysed using STATA 10 (STATA Corporation, College Station, TX USA) and EpiInfo version 6 software. The difference in the diagnostic yield was calculated using a two-sample test of proportion. Statistical tests included the two-group test of proportions, the chi-square test and the Wilcoxon rank-sum test. Statistical tests were two-sided at $\alpha=$ $0 \cdot 05$.

\section{RESULTS}

\section{Study Population}

There were 674 children enrolled, of whom 535 had an Xpert and culture result from at least one paired IS and NPA; 396 had two paired IS and NPA specimens, Figure 1. Almost a quarter, 117 (21.9\%), were HIV-infected; most had moderate or severe immune suppression, Table 1 . The median (IQR) age was 19 (11.2-38.3) months with an age range of 1.5-154 months; many children had nutritional impairment [median (IQR) weight for age $\mathrm{z}$ score $-1.4(-2.3$ to -0.5$)$ ], Table 1 . There were 87 (16.3\%) children with definite tuberculosis, 255 (47.6\%) with possible tuberculosis, and 193 (36.1\%) with no tuberculosis, Figure 1. The proportion with definite tuberculosis was similar amongst HIV-infected $(15 / 117,12.8 \%)$ and uninfected children $(72 / 417,17.3 \%) P=.25$

\section{Performance of Xpert and Smear Microscopy on 1 Paired NPA and IS Specimen}

Amongst 535 children with at least 1 paired IS and NPA specimen, the number with a positive smear, Xpert test, or culture was $30(5.6 \%), 81$ (15.1\%), and 87 (16.3\%), respectively. There was at least one positive Xpert test in $70 / 87$ (80.5\%) children with definite tuberculosis, 10/255 (3.9\%) with possible tuberculosis, and 1/193 (0.5\%) children without tuberculosis, Figure 1. The culture yield from children with definite or possible tuberculosis was $87 / 342(25.4 \%)$. The culture yield from IS (84/87, 96.6\%, 95\% CI: 92.6-100) was higher than that from NPA $(61 / 87,70.1 \%, 95 \% \mathrm{CI}: 60.3-79.9), P<.001$. Using culture as the reference, the sensitivity of smear $(30 / 87$, 34.5\%, 95\% CI: 24.3-44.7) was lower than Xpert testing (70/ 87, 80.5\%, 95\% CI: 72.0-89.0, $P<.001)$. Xpert testing detected all smear-positive cases (30/30, 100\%, 95\% CI: 88.4-100) and 40/57 (70.2\%, 95\% CI: 57.9-82.4) smear-negative cases.

\section{Performance of Xpert and Smear Microscopy on 2 Paired NPA} and IS Specimens

Amongst 396 children with 2 paired specimens, there were 63 culture-confirmed cases [60/63, 95.2\%, 95\% CI: 89.9-100 for IS and 48/63, 76.2\%, 95\% CI: $65.4-87.0$ for NPA, $P=.002$ ]; 3 children were culture-negative on IS specimens, but -positive when NPA was used. Using culture from any specimen as the reference, the sensitivity of two Xpert tests on IS (45/63, $71.4 \%$, 95\% CI: $60.0-82.9)$ was similar to that obtained with two NPAs (41/63, 65.1\%, 95\% CI: 53.0-77.2, $P=.44)$, Tables 2 and 3. Using both IS and NPA specimens, the sensitivity of Xpert testing increased to 51/63 (81.0\%, 95\% CI: 71.0-90.9). Xpert detected more than twice as many definite cases as smear microscopy on IS samples $(21 / 63,33.3 \%, 95 \%$ CI: $21.4-$ 45.3), Table 2, and on NPA samples $(16 / 63,25.4 \%, 95 \% \mathrm{CI}$ : 14.3-36.4), Table 3.

Using culture and Xpert results from NPAs only, two Xpert tests detected 41/48 (85.4\%, 95\% CI: 75.1-95.8) cultureconfirmed cases, including all 16 smear-positive and 25/32 (78.1\%, 95\% CI: 63.0-93.3) smear-negative cases. Similarly, for cases confirmed using IS, two Xpert tests detected all smear-positive (21/21, 100\%, 95\% CI: 83.9-100) and 24/39 (61.5\%, 95\% CI: 45.6-77.5) smear-negative cases. The sensitivity of Xpert for IS was higher but not significantly different in HIV-infected compared to HIV-uninfected children with definite tuberculosis $(7 / 8,87.5 \%, 95 \%$ CI: $57.9-100 \%$ vs $38 / 55$, 69.1\%, 95\% CI: 56.5-81.7, $P=.28$ ), Table 2. A similar pattern occurred with NPAs where Xpert detected 8/8 (100\%, 95\% CI: 63.1-100) HIV-infected vs 33/55 (60.0\%, 95\% CI: 46.6-73.4) HIV-uninfected children with culture-confirmed tuberculosis, $P=.46$, Table 3 . However, a higher proportion of HIV-infected than uninfected children were smear-positive [ $9 / 15$ (60\%, 95\% CI: 31.9-88.1) vs 21/72 (29.2\%, 95\% CI: 18.4-39.9)].

A second IS specimen increased the yield from Xpert by 9 cases (25\%) from 36 to 45 cases; the incremental yield of a second IS culture was 9 cases (17.6\%), from 51 to 60 cases. Amongst these, 93/396 (23\%) had a second IS and NPA taken on the same day. An incremental yield from the second IS culture on Xpert only occurred when taken on sequential days. A second NPA increased the yield from Xpert by 11 cases $(36.7 \%)$, from 30 to 41 cases, while the incremental yield from a second NPA culture was 10 cases (26.3\%), from 38 to 48 cases. The incremental yield from a second NPA culture taken on the same day was $3 / 38(8 \%)$ cases, while the yield from an NPA taken on sequential days was $7 / 38$ (18.3\%). The incremental yield from a second NPA Xpert test performed on the same day was $3 / 30(10 \%)$ cases and $8 / 30(26.7 \%)$ cases from a sequential day NPA Xpert test.

The specificity of the Xpert test was 99.1\% (95\% CI: 98.1$100)$ and $98.2 \%$ (96.8-99.6) for IS and NPA respectively, Tables 2 and 3. Ten children classified as having "Possible 


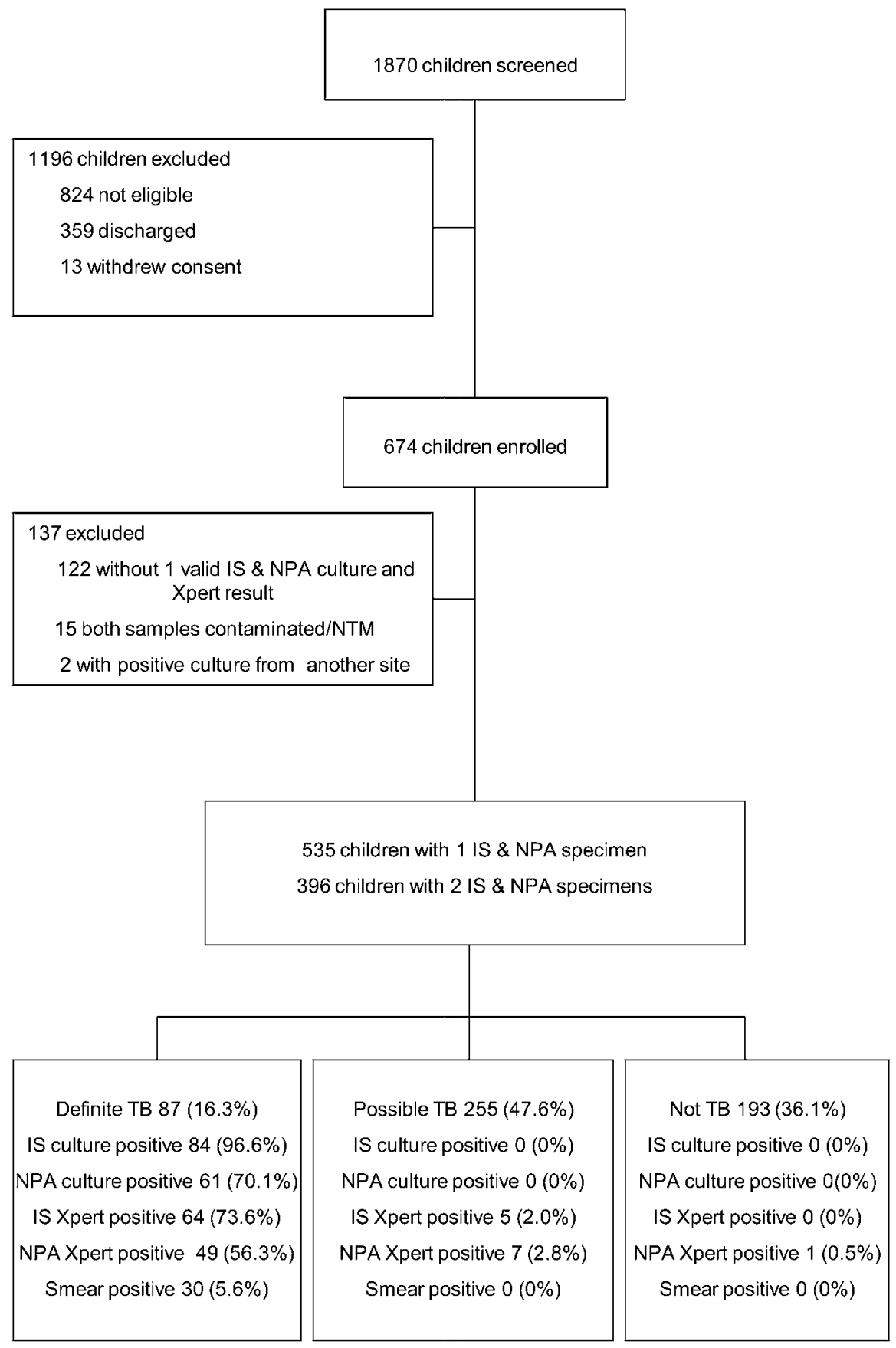

Figure 1. Study profile. Abbreviations: IS, induced sputum; NPA, nasopharyngeal aspirate; NTM, non-tuberculosis mycobacterium; TB, tuberculosis.

tuberculosis" had a positive Xpert test and negative culture, Figure 1; two had a single (negative) culture while 9 were treated for PTB with good clinical response. Xpert was positive in only $1(0.5 \%)$ of the "Not tuberculosis" group, with a weakly positive Xpert test on one NPA. Excluding the possible tuberculosis group whose Xpert tests may represent true positive cases, the specificity of Xpert was $99.5 \%$ (95\% CI: 98.4$100)$. The specificity of smear microscopy was $100 \%$.
In cases where the results for RIF susceptibility testing were available from culture (followed by MTBDRplus) and Xpert, 119/127 RIF-susceptible isolates were correctly identified by Xpert (114 RIF-sensitive and 5 RIF-resistant for both MTBDRplus and Xpert testing, Table 4). Four tests were indeterminate by Xpert but RIF-sensitive by MTBDRplus testing; 2 tests were RIF-sensitive by Xpert and inconclusive by MTBDRplus testing, 1 test was RIF-resistant by Xpert and 
Table 1. Baseline Characteristics of the Children in the Study

\begin{tabular}{|c|c|c|c|c|}
\hline & All & Definite TB & Possible TB & Not TB \\
\hline Age (months; median and IQR) & $19.0(11.2-38.3)$ & $21.1(11.9-45.7)$ & $17.9(10.6-35.0)$ & $19.9(11.2-38.4)$ \\
\hline Male n (\%) & $294(55.0)$ & $51(58.6)$ & $137(53.7)$ & $106(53.7)$ \\
\hline HIV infection $\mathrm{n}(\%)$ & $117(21.9)$ & $15(17.2)$ & $64(25.1)$ & $38(19.7)$ \\
\hline \multicolumn{5}{|l|}{ HIV WHO clinical staging $\mathrm{n}(\%)$} \\
\hline Stage 1 & $10(8.6)$ & $0(0)$ & $5(7.8)$ & $5(13.2)$ \\
\hline Stage 2 & 35 (29.9) & $5(33.3)$ & $14(21.9)$ & $16(42.1)$ \\
\hline Stage 3 & 42 (35.9) & $6(40.0)$ & $23(35.9)$ & $13(34.2)$ \\
\hline Stage 4 & $30(25.6)$ & $4(26.7)$ & $22(34.4)$ & $4(10.5)$ \\
\hline \multicolumn{5}{|l|}{ HIV CDC immune category $\mathrm{n}(\%)$} \\
\hline None & $23(22.1)$ & $2(15.4)$ & $15(26.8)$ & $6(17.1)$ \\
\hline Moderate & $35(33.7)$ & $8(61.5)$ & $16(28.6)$ & $11(31.4)$ \\
\hline Severe & $46(44.2)$ & $3(23.1)$ & $25(44.6)$ & $18(51.4)$ \\
\hline History of prior tuberculosis N (\%) & $56(10.4)$ & $6(6.9)$ & $25(9.8)$ & $25(13.0)$ \\
\hline Radiological changes suggestive of TB n (\%) & $333(67.4)$ & $55(73.3)$ & $159(68.00)$ & $119(65.0)$ \\
\hline Commenced on anti-tuberculosis treatment $\mathrm{n}(\%)$ & $283(52.9)$ & $87(100)$ & $194(76.1)$ & $0(0)$ \\
\hline \multicolumn{5}{|l|}{ Z scores (median and IQR) } \\
\hline HAZ & $-1.4(-2.5$ to -0.4$)$ & $-1.4(-2.6$ to -0.5$)$ & $-1.6(-2.7$ to -0.6$)$ & $-1.3(-2.2$ to -0.2$)$ \\
\hline WAZ & $-1.4(-2.3$ to -0.5$)$ & $-1.6(-2.7$ to -0.7$)$ & $-1.6(-2.4$ to -0.7$)$ & $-1.2(-2.1$ to -0.3$)$ \\
\hline WHZ & $-0.59(-1.5-0.3)$ & $-1.0(-2.2$ to -0.3$)$ & $-0.5(-1.5-0.6)$ & $-0.4(-1.2-0.4)$ \\
\hline Malnutrition (WAZ score <- 2) n (\%) & $68(15.6)$ & $17(29.3)$ & $32(15.6)$ & $19(11.0)$ \\
\hline Tuberculin skin-positive $\mathrm{n}(\%)$ & $191(39.2)$ & $62(74.7)$ & $110(48.3)$ & $19(10.8)$ \\
\hline
\end{tabular}

Abbreviations: CDC, Centers for Disease Control and Prevention; HAZ, height for age Z-score; HIV, human immunodeficiency virus; IQR, interquartile range; TB, tuberculosis; WAZ, weight for age Z-score; WHO, World Health Organization; WHZ, weight for height Z-score.

\section{Table 2. Accuracy of Xpert Mycobacterium tuberculosis/Rifampicin and Smear on Induced Sputum Compared With Liquid Culture}

\begin{tabular}{|c|c|c|c|c|c|c|}
\hline & Sensitivity & Specificity & PPV & NPV & $\begin{array}{l}\text { Sensitivity for Smear- } \\
\text { Positive Samples }\end{array}$ & $\begin{array}{l}\text { Sensitivity for Smear- } \\
\text { Negative Samples }\end{array}$ \\
\hline \multicolumn{7}{|c|}{ All children with complete results from at least one IS and NPA specimen $(n=535)$} \\
\hline \multicolumn{7}{|l|}{ Xpert } \\
\hline All & 64/87, $73.6(64.1-83.0)$ & $443 / 448,98.9$ (97.9-99.9) & 92.8 & 95.1 & 29/30, $96.7(89.9-100)$ & $35 / 57,61.4(48.4-74.4)$ \\
\hline $\begin{array}{l}\text { HIV infected } \\
\quad(n=117)\end{array}$ & 14/15, $93.3(79.0-100)$ & 102/102, $100(96.4-100)$ & 100 & 99.0 & $9 / 9,100(66.4-100)$ & $5 / 6,83.3(40.5-100)$ \\
\hline $\begin{array}{l}\text { HIV uninfected } \\
(\mathrm{n}=417)\end{array}$ & $50 / 72,69.4(58.5-80.3)$ & $340 / 345,98.6(97.3-99.8)$ & 90.9 & 93.9 & 20/21, $95.2(85.3-100)$ & $30 / 51,58.8(44.8-72.8)$ \\
\hline Smear microscopy & $28 / 87,32.2(22.2-42.2)$ & 448/448, 100 (99.2-100) & 100 & 88.4 & & \\
\hline \multicolumn{7}{|c|}{ Children with complete results from two paired IS and NPA specimens $(n=396)$} \\
\hline $\begin{array}{l}\text { Xpert (first IS } \\
\text { specimen) }\end{array}$ & $36 / 63,57.1(44.6-69.7)$ & 332/333, $99.7(99.1-100)$ & 97.3 & 92.5 & $21 / 21,100(83.9-100)$ & $15 / 42,35.7(20.6-50.8)$ \\
\hline \multicolumn{7}{|c|}{ Xpert (both IS specimens) } \\
\hline All & $45 / 63,71.4(60.0-82.9)$ & 330/333, $99.1(98.1-100)$ & 93.8 & 94.8 & $21 / 21,100(83.9-100)$ & $24 / 42,57.1(41.5-72.8)$ \\
\hline $\begin{array}{l}\text { HIV infected } \\
(\mathrm{n}=80)\end{array}$ & $7 / 8,87.5(57.9-100)$ & 72/72, 100 (95.0-100) & 100 & 98.6 & $6 / 6,100(54.1-100)$ & $1 / 2,50(-5.9-6.9)$ \\
\hline $\begin{array}{l}\text { HIV uninfected } \\
\quad(\mathrm{n}=315)\end{array}$ & $38 / 55,69.1(56.5-81.7)$ & 257/260, $98.8(97.6-100)$ & 92.7 & 93.8 & 15/15, $100(78.2-100)$ & $23 / 40,57.5(41.5-73.5)$ \\
\hline $\begin{array}{l}\text { Smear } \\
\text { microscopy } \\
\text { (two smears) }\end{array}$ & 21/63, 33.3 (21.4-45.3) & 333/333, 100 (99.0-100) & 100 & 88.8 & & \\
\hline
\end{tabular}

Data are the number correct/number tested, \% (95\% confidence interval).

Abbreviations: HIV, human immunodeficiency virus; IS, induced sputum; NPA, nasopharyngeal aspirate; NPV, negative predictive value; PPV, positive predictive value. 
Table 3. Accuracy of Xpert Mycobacterium tuberculosis/Rifampicin and Smear on Nasopharyngeal Aspirates Compared With Liquid Culture

\begin{tabular}{|c|c|c|c|c|c|c|}
\hline NPA & Sensitivity & Specificity & PPV & NPV & $\begin{array}{l}\text { Sensitivity for Smear- } \\
\text { Positive Samples }\end{array}$ & $\begin{array}{l}\text { Sensitivity for Smear- } \\
\text { Negative Samples }\end{array}$ \\
\hline \multicolumn{7}{|c|}{ All children with complete results from at least one IS and NPA specimen $(n=535)$} \\
\hline \multicolumn{7}{|l|}{ Xpert } \\
\hline All & $49 / 87,56.3(45.7-67.0)$ & $440 / 448,98.2(97.0-99.5)$ & 86.0 & 92.1 & $27 / 30,90.0(78.6-100)$ & $22 / 57,38.6(25.6-51.6)$ \\
\hline $\begin{array}{l}\text { HIV infected } \\
(n=117)\end{array}$ & $10 / 15,66.7(39.6-93.7)$ & 100/102, $98.0(95.3-100)$ & 83.0 & 95.2 & $8 / 988.9,(63.2-100)$ & 2/6 33.3, (0-87.5) \\
\hline $\begin{array}{l}\text { HIV } \\
\quad \text { uninfected } \\
(n=417)\end{array}$ & $39 / 72,54.2(42.4-66.0)$ & 339/345, $98.3(96.9-99.6)$ & 91.1 & 86.7 & 19/21, $90.5(76.8-100)$ & 20/51, $39.2(25.3-53.1)$ \\
\hline Smear & $21 / 87,24.1(15.0-33.3)$ & $448 / 448,100(99.2-100)$ & 100 & 87.2 & & \\
\hline \multicolumn{7}{|c|}{ Children with complete results from two paired IS and NPA specimens $(n=396)$} \\
\hline $\begin{array}{l}\text { Xpert } \\
\text { (first NPA } \\
\text { specimen) }\end{array}$ & $30 / 63,47.6(34.9-60.3)$ & 329/333, $98.8(97.6-100.0)$ & 88.2 & 90.9 & 15/16, $93.8(80.4-100)$ & $15 / 47,31.9(18.1-45.7)$ \\
\hline \multicolumn{7}{|c|}{ Xpert (both NPA specimens) } \\
\hline All & $41 / 63,65.1(53.0-77.2)$ & 327/333, 98.2 (96.8-99.6) & 87.2 & 93.7 & 16/16, $100(79.4-100)$ & $25 / 47,53.2(38.4-68.0)$ \\
\hline $\begin{array}{l}\text { HIV infected } \\
(\mathrm{n}=80)\end{array}$ & $8 / 8100,(63.1-100)$ & 70/72, $97.2(93.3-100)$ & 80 & 100 & $4 / 4,100(39.8-100)$ & $4 / 4,100(39.8-100)$ \\
\hline $\begin{array}{l}\text { HIV } \\
\text { uninfected } \\
(n=315)\end{array}$ & $33 / 55,60.0(46.6-73.4)$ & 256/260, 98.5 (97.0-100.0) & 89.2 & 92.1 & $12 / 12,100(73.5-100)$ & $21 / 43,48.8(33.3-64.4)$ \\
\hline $\begin{array}{l}\text { Smear (two } \\
\text { smears) }\end{array}$ & $16 / 63,25.4(14.3-36.4)$ & $333 / 333,100(98.9-100)$ & 100 & 87.7 & & \\
\hline
\end{tabular}

Data are the number correct/number tested, \% (95\% confidence interval).

Abbreviations: HIV, human immunodeficiency virus; IS, induced sputum; NPA, nasopharyngeal aspirate; NPV, negative predictive value; PPV, positive predictive value.

RIF-sensitive by MTBDRplus testing, and 1 test was RIFsensitive by Xpert and RIF-resistant by MTBDRplus. In discordant cases, phenotypic susceptibility testing confirmed the result from MTBDRplus testing. On a per specimen analysis, the sensitivity and specificity of Xpert for the diagnosis of RIF resistance were $83.3 \%$ and $99.1 \%$, respectively. Xpert testing provided faster results than culture (0 [IQR 0-3] vs 15 days [IQR 12-20], $P<.001$ ).

\section{DISCUSSION}

This study has shown that the Xpert testing of two NPA specimens detected almost two thirds of culture-confirmed

Table 4. Detection of Rifampicin Resistance by Xpert- and Culture/MTBDRplus-Based Drug Susceptibility Testing (Per Specimen Analysis)

\begin{tabular}{lccc}
\hline & $\begin{array}{c}\text { Resistant } \\
\text { (MTBDRplus) }\end{array}$ & $\begin{array}{c}\text { Sensitive } \\
\text { (MTBDRplus) }\end{array}$ & $\begin{array}{c}\text { Inconclusive } \\
\text { (MTBDRplus) }\end{array}$ \\
\hline Resistant (Xpert) & 5 & 1 & 0 \\
Sensitive (Xpert) & 1 & 114 & 2 \\
Indeterminate (Xpert) & 0 & 4 & 0 \\
\hline
\end{tabular}

tuberculosis in young children hospitalized with suspected PTB, with very high specificity. The number of cases detected by Xpert testing on repeat NPAs was similar to that which was obtained for two IS specimens, and was more than double that which was detected by smear microscopy. A single Xpert test on an NPA detected almost all smear-positive cases. This study contributes further evidence for the usefulness of Xpert testing in diagnosing childhood tuberculosis and in recommending that smear microscopy be replaced. This is also the first study to show the usefulness of Xpert using NPAs.

The yield from Xpert testing on NPAs represents an important potential advance in the diagnosis of childhood tuberculosis. The sensitivity obtained with NPAs was higher than reported for other nucleic acid amplification tests [8,9], possibly due to the improved accuracy of Xpert testing [10]. Performing Xpert testing on both IS and NPAs detected $>80 \%$ of culture-confirmed cases; however, obtaining four specimens is likely to be too costly and labour-intensive to be feasible in resource-poor settings. Microbiologic diagnosis in children has been hampered by the inability to obtain IS samples and by paucibacillary disease, making smear a poor tool for diagnosis. Healthcare workers have not been widely trained to obtain IS samples, and facilities for induction frequently do 
not exist [5]. NPAs may therefore enable diagnosis, as specimens can be easily and non-invasively obtained. Our findings are consistent with other pediatric studies from Uganda, Yemen and Peru, reporting that NPAs may be useful for diagnosis $[8,9,14,15]$. These studies, using culture and PCR on NPA and other specimens, have shown an appreciable yield similar or less than that from IS or GA. In contrast to our study, these studies enrolled older children (median age 3-5 years), had small sample sizes, did not repeat NPAs, often did not undertake IS, and did not use Xpert testing.

Similar to the Xpert results for IS [6], two NPA specimens were necessary to detect most definite cases. The increased sensitivity from a second specimen may be due to the low bacillary load in children, as evidenced by most cases exhibiting smear-negative disease. A second specimen was especially valuable when obtained the following day, presumably allowing sufficient time for respiratory secretions containing mycobacteria to accumulate. Although WHO recommends a single Xpert test for tuberculosis suspects, our findings indicate that a second test either on an NPA or on an IS sample should be recommended in children.

Approximately a third of the children had cultureconfirmed tuberculosis, but negative Xpert tests. In these children, IS provided a higher yield for culture than NPAs. A study from Yemen also showed the superior sensitivity of IS over NPA specimens for culture [14]. In several studies including some in primary care settings, sputum induction has been shown to be safe, effective, and feasible for the diagnosis of PTB in young children [3-6, 9]. Increasing availability and improved affordability of Xpert tests enables the rapid confirmation of tuberculosis and rifampicin resistance close to the point of care. Therefore, upscaling the capacity to obtain IS samples in children should be a priority. However, in the absence of such capacity and in settings where culture is not possible, Xpert testing of two NPAs should be recommended as the first-line investigation for children with suspected PTB.

Xpert results were available on the same day, which was a much shorter period of time than that required for culture. A lack of treatment or delayed initiation of therapy remains a major challenge, with studies reporting that $20 \%$ to $47 \%$ of children with culture-confirmed tuberculosis are discharged from hospitals without commencing tuberculosis therapy [16-18]. Disease progression may occur rapidly, and tracing children after discharge may be difficult [16]. The use of Xpert enables the prompt initiation of a therapy, and minimizes the number of children who may go untreated or receive delayed treatment.

Approximately half of the children were diagnosed with possible tuberculosis, of whom $76 \%$ were treated based on clinical diagnosis. Children who were not treated and who were lost to follow-up were included in the possible tuberculosis group, as the response in the absence of tuberculosis treatment could not be evaluated. A minority of those with possible tuberculosis were Xpert-positive; these probably represent true cases, as almost all were treated for tuberculosis with good clinical response [19]. A better diagnostic test to distinguish those children who have tuberculosis amongst the possible tuberculosis cases is urgently needed. However, the absence of a reference standard other than culture makes the evaluation of any new test in children with possible tuberculosis challenging.

The limitations of this study include the small numbers of HIV-infected children with culture-confirmed disease, few rifampicin-resistant cases, and the need to split the NPA and sputum sediment between culture and Xpert testing. We split NPA and sputum pellets to directly compare culture and Xpert testing on the same specimen. Although Xpert is designed for use directly on specimens, adult studies have demonstrated an equivalent performance when the testing has been carried out directly on a specimen or on a pellet [10]. We did not compare GLs, as several studies have demonstrated the superiority of IS over GL for mycobacterial culture in hospitalized children with suspected tuberculosis [3-5, 20-22]. The generalizability of the results to children with less severe illness requires further study. Furthermore, cost efficacy analyses of Xpert are needed. However, a recent study suggests that the replacement of smear microscopy with Xpert testing in adults is highly cost-effective [23]. Xpert testing may be especially suited to resource-constrained settings where culture is unlikely to be routinely available due to cost and the complexity of mycobacterial culture.

\section{CONCLUSION}

Xpert provides a useful test for the rapid diagnosis of tuberculosis in children on repeated NPA or IS specimens. The preferred sample for culture confirmation is IS; however, the capacity for IS collection and testing in children must be increased. Xpert testing on two NPAs should be recommended for children in settings where IS collection and mycobacterial culture are not possible.

\section{Notes}

Acknowledgments. We thank the National Health Laboratory Service at Groote Schuur Hospital for their microbiology diagnostic services, the children who participated in the study, the children's caregivers, and the staff at the Red Cross War Memorial Children's Hospital and the New Somerset Hospital for their support.

Financial support. This work was supported by the National Institutes of Health, USA (grant number 1R01HD058971-01), the National Health Laboratory Services Research Trust, the Medical Research Council of South Africa, and The Wellcome Trust (grant number 085251/B/08/Z).

Potential conflicts of interest. M. P. N. has received funding from the Foundation for Innovative New Diagnostics (FIND, Geneva, Switzerland) 
to assess the performance and effect of Xpert. C. C. B. is employed by FIND. FIND is a non-profit organization that collaborates with industry partners, including Cepheid (the manufacturers of Xpert), on the development, assessment, and demonstration of new diagnostic tests. All other authors report no potential conflicts.

All authors have submitted the ICMJE Form for Disclosure of Potential Conflicts of Interest. Conflicts that the editors consider relevant to the content of the manuscript have been disclosed.

\section{References}

1. Cuevas LE. The urgent need for new diagnostics for symptomatic tuberculosis in children. Indian J Pediatr 2011; 78:449-55.

2. Connell TG, Zar HJ, Nicol MP. Advances in the diagnosis of pulmonary tuberculosis in $\mathrm{HIV}$-infected and HIV-uninfected children. J Infect Dis 2011; 204:S1151-58.

3. Zar HJ, Hanslo D, Apolles P, Swingler G, Hussey G. Induced sputum versus gastric lavage for microbiological confirmation of pulmonary tuberculosis in infants and young children: a prospective study. Lancet 2005; 365:130-4.

4. Iriso R, Mudido PM, Karamagi C, Whalen C. The diagnosis of childhood tuberculosis in an HIV-endemic setting and the use of induced sputum. Int J Tuberc Lung Dis 2005; 9:716-26.

5. Moore A, Apolles P, de Villiers PJT, Zar HJ. Sputum induction for diagnosis of childhood pulmonary tuberculosis (PTB) in a community setting. Int J Tuber Lung Dis 2011; 15:1185-90.

6. Nicol MP, Workman L, Isaacs W, et al. Accuracy of the Xpert MTB/RIF test for the diagnosis of pulmonary tuberculosis in hospitalized children in a high HIV-prevalence area. Lancet Infect Dis 2011; 11:819-24.

7. WHO. Automated Real-time Nucleic Acid Amplification Technology for Rapid and Simultaneous Detection of Tuberculosis and Rifampicin Resistance: Xpert MTB/RIF System. Policy Statement. 2011. Geneva: World Health Organisation, 2011. Available at: http://www.who.int/ tb/. Accessed 14 May 2012.

8. Oberhelman RA, Soto-Castellares G, Gilman RH, et al. Diagnostic approaches for paediatric tuberculosis by use of different specimen types, culture methods, and PCR: a prospective case-control study. Lancet Infect Dis 2010; 10:612-20.

9. Owens S, Abdel-Rahman IE, Balyejusa S, et al. Nasopharyngeal aspiration for diagnosis of pulmonary tuberculosis. Arch Dis Child 2007; 92:693-6.

10. Boehme CC, Nabeta P, Hillemann D, et al. Rapid molecular detection of tuberculosis and rifampin resistance. N Engl J Med 2010; 363:1005-15.

11. WHO. WHO case definitions of HIV for surveillance and revised clinical staging and immunological classifi cation of HIV-related disease in adults and children, 2006. Available at: http://www.who.int/hiv/ pub/guidelines/HIVstaging150307.pdf. Accessed 5 October 2011.

12. Caldwell MB, Oxtoby MJ, Simonds RJ, Lindegren ML, Rogers MF. 1994 revised classification system for human immunodeficiency virus infection in children less than 13 years of age. Centers for Disease Control and Prevention. MMWR 1994; 43:1-10.

13. Barnard M, Albert H, Coetzee G, O’Brien R, Bosman ME. Rapid molecular screening for multidrug-resistant tuberculosis in a high-volume public health laboratory in South Africa. Am J Respir Crit Care Med 2008; 177:787-92.

14. Al-Aghbari N, Al-Sonboli N, Yassin MA, et al. Multiple sampling in one day to optimize smear microscopy in children with tuberculosis in Yemen. PLoS One 2009; 4:e5140.

15. Franchi LM, Cama RI, Gilman RH, Montenegro-James S, Sheen P. Detection of Mycobacterium tuberculosis in nasopharyngeal aspirate samples in children. Lancet 1998; 352:1681-2.

16. Moore DP, Klugman KP, Madhi SA. Role of Streptococcus pneumoniae in hospitalization for acute community-acquired pneumonia associated with culture-confirmed Mycobacterium tuberculosis in children: a pneumococcal conjugate vaccine probe study. Pediatr Infect Dis J 2010; 29:1099-104.

17. Engelbrecht AL, Marais BJ, Donald PR, Schaaf HS. A critical look at the diagnostic value of culture-confirmation in childhood tuberculosis. J Infect 2006; 53:364-9.

18. Jeena PM, Pillay P, Pillay T, Coovadia HM. Impact of HIV-1 co-infection on presentation and hospital-related mortality in children with culture proven pulmonary tuberculosis in Durban, South Africa. Int J Tuberc Lung Dis 2002; 6:672-8.

19. Newton SM, Brent AJ, Anderson S, Whittaker E, Kampmann B. Paediatric tuberculosis. Lancet Infect Dis 2008; 8:498-510.

20. Zar HJ, Tannenbaum E, Apolles P, Roux P, Hanslo D, Hussey G. Sputum induction for the diagnosis of pulmonary tuberculosis in infants and young children in an urban setting in South Africa. Arch Dis Child 2000; 82:305-8.

21. Singh M, Mynak ML, Kumar L, Mathew JL, Jindal SK. Prevalence and risk factors for transmission of infection among children in household contact with adults having pulmonary tuberculosis. Arch Dis Child 2005; 90:624-8.

22. Uriyo J, Gosling RD, Maddox V, et al. Prevalences of Pneumocystis jiroveci, Mycobacterium tuberculosis and Streptococcus pneumoniae infection in children with severe pneumonia, in a tertiary referral hospital in northern Tanzania. Ann Trop Med Parasitol 2006; 100:245-9.

23. Vassall A, van Kampen S, Sohn H, et al. Rapid Diagnosis of Tuberculosis with the Xpert MTB/RIF Assay in High Burden Countries: a Cost-Effectiveness Analysis. PLoS Med 2011; 8:e100112. 